\title{
Pulmonary vascular disease in neonates with transposition of the great arteries and intact ventricular septum
}

\author{
Anil Kumar, Glenn P Taylor, George G S Sandor, Michael W H Patterson
}

\begin{abstract}
Background-Progressive pulmonary vascular disease in surgically unrepaired transposition of the great arteries with or without ventricular septal defect had been frequently described in the past. Occurrence of progressive pulmonary vascular disease has been reported even after atrial switch procedure done at three months of age. With the advent of neonatal surgical repair, this problem is virtually non-existent. There is a small subgroup of infants with transposition of the great arteries who show pulmonary vascular disease in the neonatal period that can adversely affect the surgical outcome. The clinico-pathological correlation in this group of patients was studied. Observations-Three patients, with transposition of the great arteries and intact ventricular septum, who showed histological evidence of pulmonary vascular disease in the neonatal period or early infancy are described. Two of these patients, continued to have poor systemic oxygenation despite adequate atrial communication. One patient had a closed ductus arteriosus within the first two hours of birth while on prostaglandin $E_{1}$ infusion.
\end{abstract}

Conclusions-In the absence of left ventricular outflow tract obstruction, a poor response to atrial septostomy suggests pulmonary hypertension and pulmonary vascular disease. Antenatal constriction of the ductus arteriosus may contribute to such changes in pulmonary vasculature.

(Br Heart $\mathcal{~} 1993 ; 69: 442-445)$

Division of Cardiology A Kumar

G G S Sandor

$M$ W H Patterson

Division of Pathology, British Columbia British Columbia
Children's Hospital, Vancouver, British Columbia, Canada G P Taylor

Correspondence to: Anil Kumar MD, University of Florida College of Medicine, Division of Pediatric Cardiology, Box 100296, JHMHC, Gainesville, Florida 326100296, USA.

Accepted for publication 4 January 1993
Progressive pulmonary vascular disease has been an often described complication of surgically unrepaired transposition of the great arteries. The vascular disease is more pronounced if transposition is associated with a ventricular septal defect or a patent ductus arteriosus. ${ }^{1}$ The abnormal pulmonary vascular changes increase with age. ${ }^{2}$ In infants less than six months of age with transposition of the great arteries, intact ventricular septum, and a closed ductus arteriosus, pulmonary vascular changes of more than grade 2 (Heath and Edwards ${ }^{3}$ ) are unusual. ${ }^{12}$ Viles, et al, reported one six month old infant in his series, with transposition of the great arteries, intact ventricular septum, and no ductus arte- riosus who had grade 3 pulmonary vascular disease. ${ }^{4}$ Ferencz included in her series of $\bar{\sigma}$ transposition of the great arteries, four infants less than six months of age with advanced lesions, the youngest being six weeks old. ${ }^{5}$ The state of the ductus arteriosus was not indicated in this series. Grade 2 lesions have been reported more often in young infants less than six months of age (Ferencz 17 of 71 , Newfeld et al, 5 of 52, Edwards and Edwards 3 of 12)..$^{512}$ Arterial switch repair done in the or first two weeks after birth theoretically eliminates the risk of progressive damage to the pulmonary vascular bed. There is, however, a small group of neonates with transposition of $z$ the great arteries and intact ventricular septum, who show intimal proliferation or more $\vec{\theta}$ advanced lesions in pulmonary vasculature in first few weeks after birth. ${ }^{516}$ The presence of pulmonary vascular lesions in this age group can jeopardise apparently successful surgical correction. We report three cases of transposition of the great arteries, without ventricular septal defect or ductus arteriosus, who had significant pulmonary vascular disease in the neonatal period that adversely affected their outcome.

\section{Patients and methods}

We reviewed all necropsies on patients with transposition of the great arteries who died at 8 less than six months old between 1986 and $\frac{3}{3}$ 1991 at our institution. Of the 11 cases, five 을 had a ventricular septal defect and six had an intact ventricular septum. The sections of the non-inflated lung, at least one section from $N$ each lobe, were studied histologically after staining with haematoxylin and eosin, 0 trichrome, and elastic stains. Formal morpho- $\mathrm{W}$ metric studies were not performed. In seven cases grade 0-1 Heath Edwards pulmonary vascular changes were present. One patient showed occasional foci of intimal proliferation. Three patients, aged six days, 10 days, and 3.5 months, showed unexpected advanced changes, and are reviewed (table).

\section{PATIENT 1}

This male infant was noted to be cyanotic $\frac{8}{0}$ and acidotic immediately after birth. He was mechanically ventilated and given an infusion $\frac{0}{7}$ of prostaglandin $\mathrm{E}_{1}$. There was no significant improvement in oxygen saturation. An echocardiogram confirmed the diagnosis of transposition of the great arteries with intact ventricular septum. There was no obstruction of the left ventricular outflow and the ductus 
Summary of clinical findings

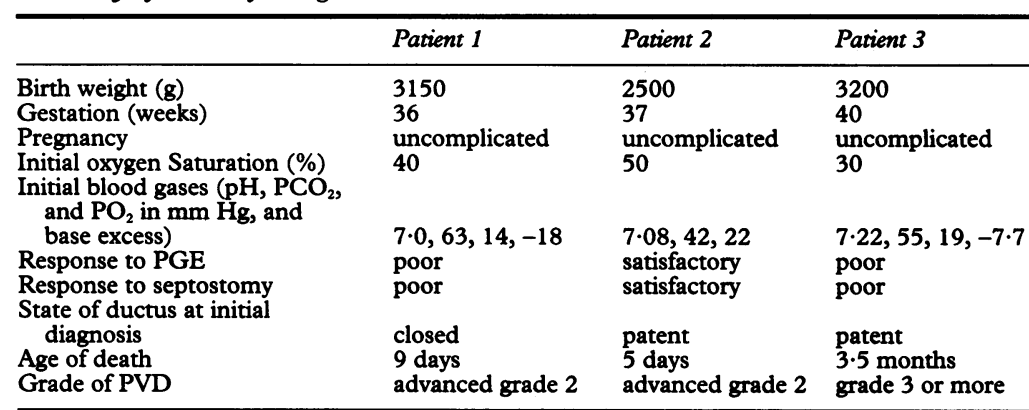

PGE, prostaglandin $E_{1} ; P V D$, pulmonary vascular disease.

was closed. Balloon atrial septostomy was done successfully through the umbilical vein, leading to transient improvement in colour and oxygenation. Oxygen saturation dropped to pre-septostomy values within the next four hours. Cardiac catheterisation performed on day 1 after birth, showed the left ventricular systolic pressure to be two thirds that of the right ventricle $(40 \mathrm{~mm} \mathrm{Hg} / 60 \mathrm{~mm} \mathrm{Hg}$ ) and main pulmonary artery pressure was $35 / 8 \mathrm{~mm}$ $\mathrm{Hg}$ with a mean of $15 \mathrm{~mm} \mathrm{Hg}$. Saturation data suggested poor effective pulmonary blood flow despite septostomy (superior vena cava $30 \%$, aorta $45 \%$, and left atrium $95 \%$ ). An aortogram showed a closed ductus while the patient was being treated with prostaglandin $\mathrm{E}_{1}$. There were no noteworthy aortopulmonary collateral vessels. He underwent Blalock Hanlon septectomy on day 2 without significant improvement in oxygenation, although acidosis improved. $\mathrm{He}$ remained clinically stable and a Mustard procedure was performed on day 9 . The patient died of uncontrolled haemorrhage from a liver laceration during surgery. Necropsy showed an anatomically satisfactory surgical repair. Lung histology showed mild medial muscular hypertrophy of the preacinar arteries and minimal muscular hypertrophy of the

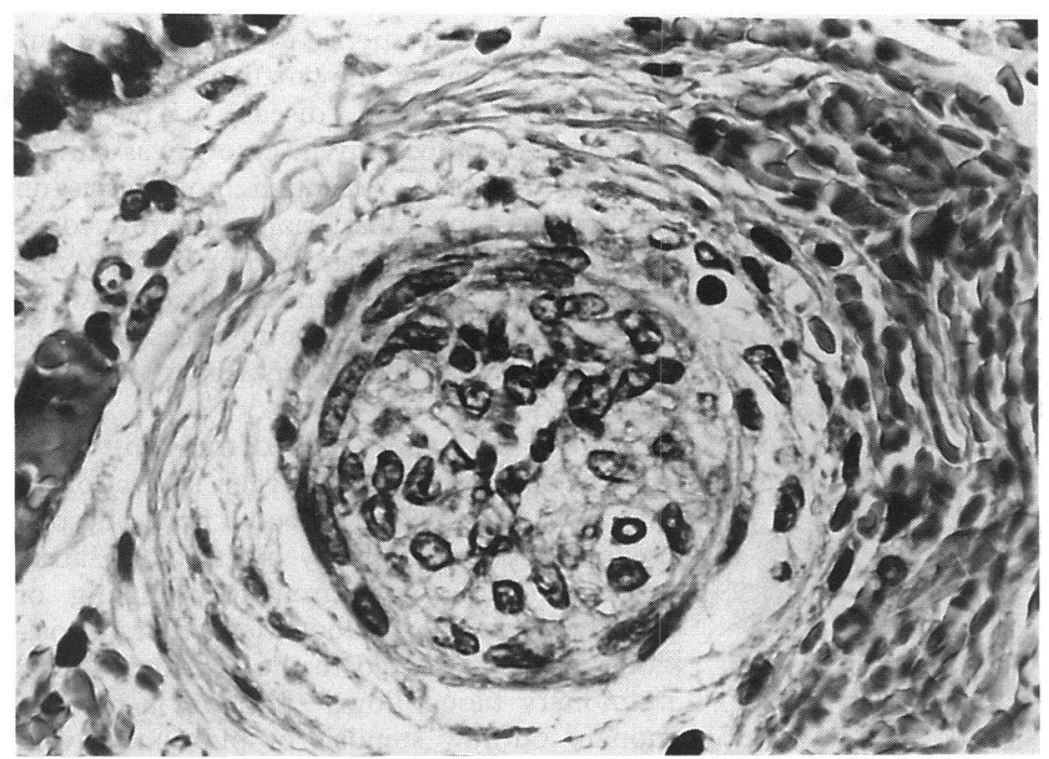

Figure 1 Patient 1, terminal bronchiole arteriole showing nearly occlusive intimal proliferation (haematoxylin and eosin; original magnification, $\times 1000$ ). intra-acinar arteries. There was however, striking intimal proliferation of the pre and intra-acinar arteries. Many vessels showed occlusion or near occlusion of their lumens (fig 1).

\section{PATIENT 2}

This boy presented with cyanosis in the first two hours after birth. Prostaglandin $\mathrm{E}_{1}$ infusion was started and he was mechanically ventilated. Oxygen saturation increased from $50 \%$ to $70 \%$ over the next hour. An echocardiogram confirmed the diagnosis of transposition of the great arteries. The ventricular septum was intact and there was no obstruction to the left ventricular outflow tract. The ductus was widely patent. Balloon atrial septostomy was performed on the first day, and led to improvement in oxygen saturation to $80 \%-90 \%$. He underwent an arterial switch operation on day 5 . Signs of persistent low cardiac output developed after surgery, and he died 24 hours later. Necropsy showed anatomically satisfactory arterial anastomoses and unobstructed coronary arteries. The myocardium did not show any histological evidence of infarction. The lung histology showed extensive occlusive intimal proliferation and mild muscular hypertrophy in the preacinar arteries. Minimal changes were present in intra-acinar arteries (fig 2).

PATIENT 3

This female infant presented with cyanosis within the first four hours of birth. An echocardiogram confirmed the diagnosis of transposition of the great arteries. The ventricular septum was intact, there was no obstruction to the left ventricular outflow tract and the ductus arteriosus was widely patent. Balloon atrial septostomy was done on the first day and resulted in initial improvement of oxygen saturation from $30 \%$ to $70 \%$. Systemic saturation decreased to preseptostomy values over the next four weeks. On day 37 a blade atrial septostomy was done with improvement in oxygen saturation to $65 \%$. Cardiac catherisation at this time showed left ventricular pressure that was half the systemic pressure $(50 v 100 \mathrm{~mm} \mathrm{Hg}$ ), and poor effective pulmonary blood flow (oxygen saturations: superior vena cava $56 \%$, right ventricle $67 \%$, left ventricle $100 \%$ ). Aortic angiography showed an absence of a ductus arteriosus or important aortopulmonary collateral vessels. By echocardiographic assessment the atrial communication was $6-7 \mathrm{~mm}$. Because of persistent cyanosis, the patient underwent Blalock Hanlon septectomy at three months of age. She remained cyanotic but clinically stable. A Mustard procedure was carried out at 3.5 months of age. She died two days after the operation with clinical evidence of pulmonary hypertension, manifested by low systemic blood pressure, low pulmonary baffle venous pressure, and high central venous pressure. Necropsy showed the venous baffles to be unobstructed. The lung histology showed pulmonary vascular disease with obliterative intimal hyperplasia 


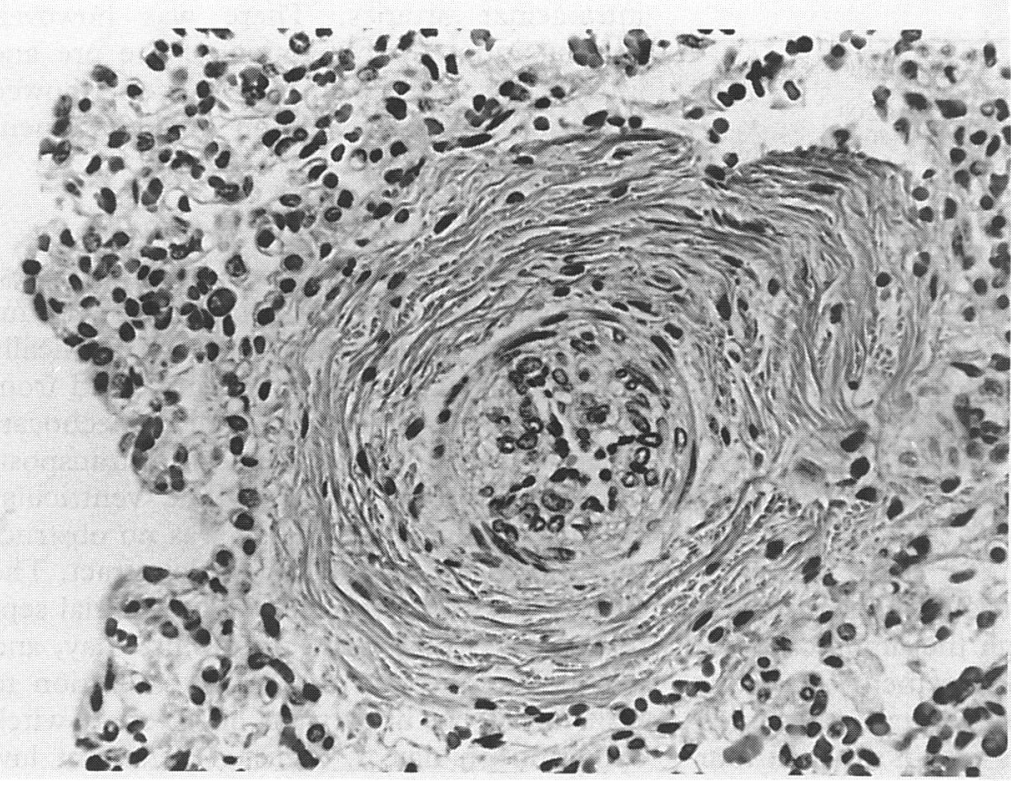

Figure 2 Patient 2, terminal bronchiole arteriole showing medial hyperplasia and intimal proliferation (haematoxylin and eosin; original magnification, $\times 500$ )

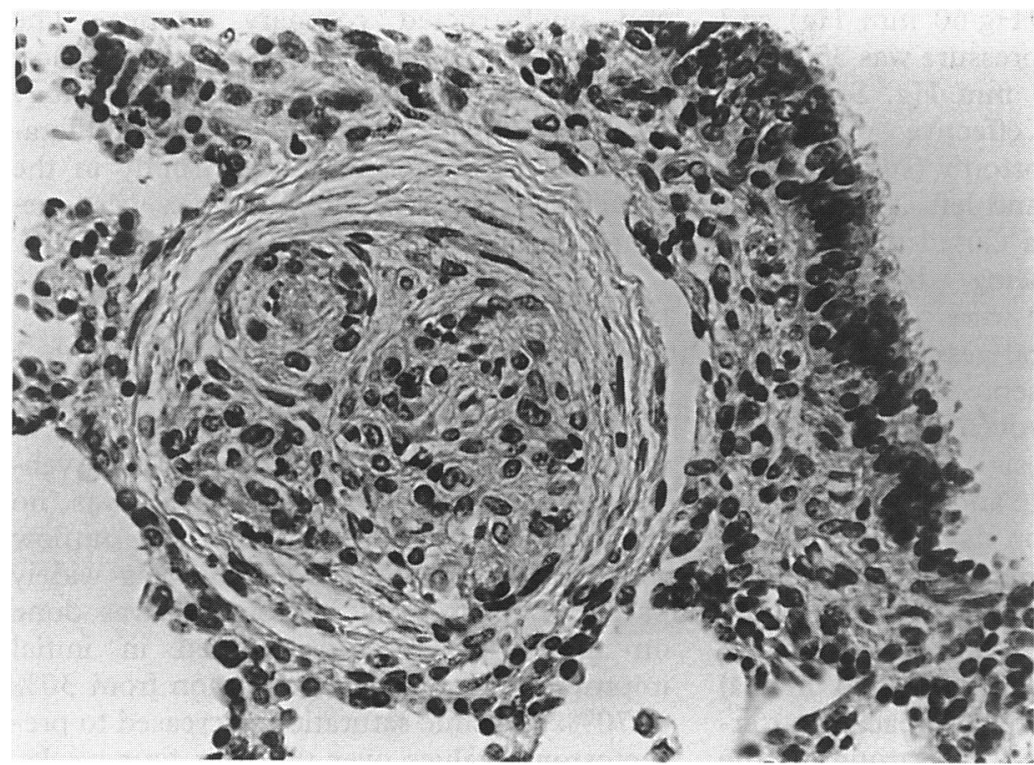

Figure 3 Patient 3, pre-acinar artery with intimal proliferation and recanalisation, (haematoxylin and eosin; original magnification, $\times 500$ )

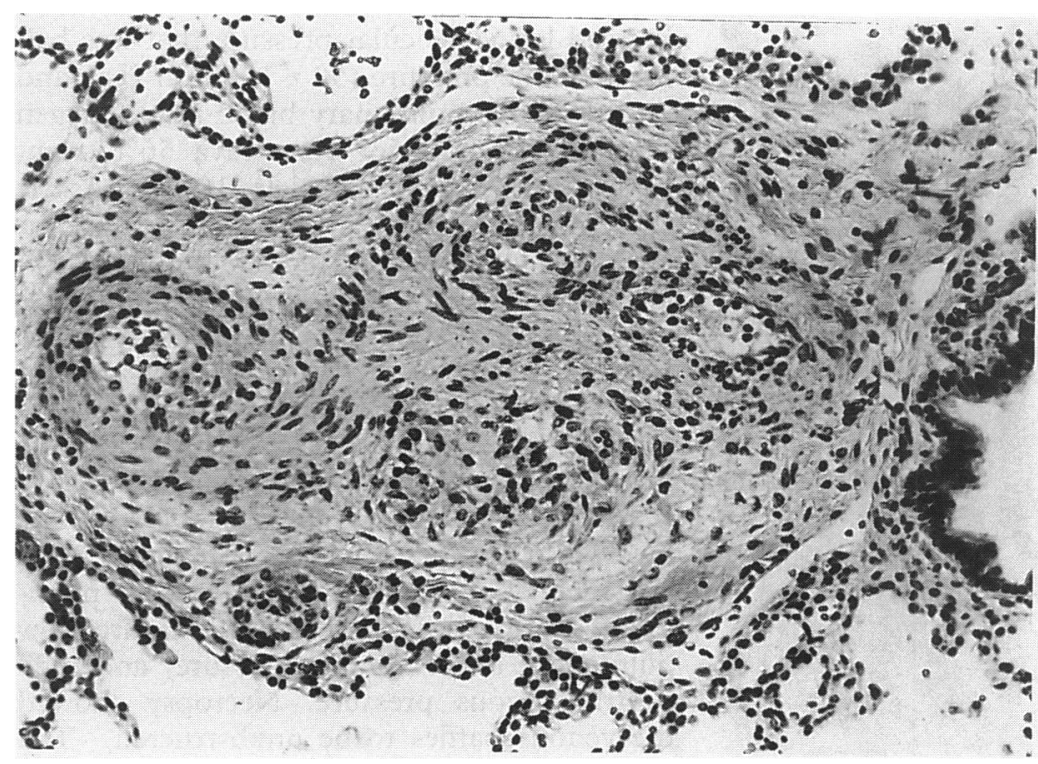

Figure 4 Patient 3, suggestive plexiform lesion of medium sized preacinar artery, (haematoxylin and eosin; original magnification, $\times 250$ ) and fibrosis, suggestive plexiform lesions, and occasional foci of fibrinoid necrosis (figs 3 and 4).

\section{Discussion}

The first two cases in our report show grade 2 changes (Heath and Edwards classification ${ }^{3}$ ) in pulmonary vasculature within first 10 days after birth, whereas the third case shows more advanced lesions at 3.5 months of age. Considerable intimal proliferation with luminal occlusion in association with transposition of the great arteries and intact ventricular septum is rarely reported in infants as young as one week. Occasional cases have been included in some reports of lung histology in cases of transposition, but because of the small numbers, its significance has not been fully appreciated.457 Even though the pulmonary vascular lesions in the first two cases are classified as only grade 2 , the findings suggest a progressive rather than reversible change. Occlusive intimal proliferation is considered to be a pre-dilatation lesion, and indicates a high risk group for persistent or progressive pulmonary hypertension. ${ }^{89}$ Severe occlusive intimal proliferation identified in the first week of life suggests that the smaller pulmonary arterioles had sustained a significant injury before birth. Development of severe pulmonary vascular disease has been reported to occur after Mustard procedure done at three months of age. ${ }^{10}$ This infant did not have either a ventricular septal defect or patent ductus arteriosus. In a report on the outcome of a neonatal arterial switch operation for transposition of the great arteries with intact ventricular septum, one infant was said to have progressive pulmonary vascular disease leading to death. ${ }^{11}$ These cases are similar to our patients in the occurrence of pulmonary vascular disease in the neonatal period. Because of the unusual presence of considerable intimal proliferation, we believe the first two neonates would have continued to show evidence of pulmonary vascular disease, had they survived their surgery. Case 3 had shown poor response to atrial septostomy and was ultimately shown to have advanced pulmonary vascular disease. The poor response to septostomy in case 1 was similar to that of case 3. Although most series on outcome of neonatal arterial switch procedure do not include any cases with pulmonary vascular disease, a larger and longer follow up may show this small subgroup of cases of pulmonary vascular disease in transposition of the great arteries.

Two of our cases showed transient or no clinical improvement in oxygen saturation or effective pulmonary blood flow despite creation of anatomically satisfactory atrial communication. Intercirculatory mixing or effective pulmonary blood flow in transposition is directly related to the amount of total pulmonary blood flow. ${ }^{12}$ If there is no pulmonary outflow stenosis or pulmonary vein stenosis, the major determinant of pulmonary vascular resistance will be the state of the 
pulmonary vascular bed. In the presence of pulmonary vascular disease the pulmonary blood flow will decrease, as will the effective pulmonary blood flow. Although poor intercirculatory mixing in transposition of the great arteries can occur because of streaming effects, ${ }^{13}$ in our cases the most important factor was the presence of pulmonary vascular disease.

The reason for such early appearance of these histological abnormalities is not clear. Circulation before birth where there is transposition is different from normal as the blood with higher partial pressure of oxygen from the umbilical vein passes through the pulmonary artery and ductus arteriosus. If the ductus arteriosus is constricted antenatally, a greater volume of blood with higher partial pressure of oxygen will flow through the immature pulmonary vasculature at higher pressure. Theoretically, this can explain the early onset of pulmonary vascular disease in some infants with transposition of the great arteries. Antenatal ductal constriction is known to be associated with persistent pulmonary hypertension in neonates. ${ }^{14}$ The typical vascular changes present in this syndrome are noticeable hypertrophy and peripheral extension of muscle in pre and intra-acinar pulmonary arteries. There is no intimal proliferation. McKenzie and Haworth noted intimal proliferation leading to luminal occlusion in a case of persistent pulmonary hypertension in a newborn infant. ${ }^{15}$ The reported histological changes were similar to our first two cases. Identification of a closed ductus arteriosus in our first case, on echocardiography and angiography while on prostaglandin $E_{1}$ infusion, lends support to this hypothesis.

In conclusion, these cases show that significant pulmonary vascular disease occurs in some cases of transposition of the great arteries soon after birth. This can adversely affect the outcome of neonatal surgical repair. The possibility of pulmonary vascular disease should be considered in those cases of transposition without left ventricular outflow obstruction, who show poor response to atrial septostomy. In these patients a lung biopsy should be considered at the time of surgical repair. After operation, they should be followed up for development of pulmonary hypertension.

We thank Dr Michael L Epstein for his helpful comments.

1 Newfeld EA, Paul MA, Muster AJ, Idriss FS. Pulmonary vascular disease in complete transposition of the grea arteries: a study of 200 patients. Am $\mathcal{F}$ Cardiol 1974 34:75-82.

2 Edwards WD, Edwards JE. Hypertensive pulmonary vascular disease in d-transposition of the great arteries. $A m$ f Cardiol 1978;41:921-4.

3 Heath D, Edwards JE. The pathology of hypertensive pulmonary vascular disease. Circulation 1958;18:533-47.

4 Viles PH, Ongley PA, Titus JL. The spectrum of pulmonary vascular disease in transposition of the great arteries. Circulation 1969;40:31-41.

5 Ferencz C. Transposition of the great vessels: pathophysiologic considerations based upon a study of lungs. Circulation 1966;33:232-42.

6 Franco Zapata JM, Suarez de Lezo J, Alemany F, Salas Franco Zapata JM, Suarez de Lezo J, Alemany F, Salas
Molina J, Martinez RC. Bronchopulmonary anastomoses and early pulmonary hypertension in complete moses and early pulmonary hypertension in complete transpositic

7 Clarkson PA, Neutze JM, Wardill JC, Baratt-Boyes BG. The pulmonary vascular bed in patients with complete transposition of the great arteries. Circulation 1976;53 539-43

8 Haworth SG. Pulmonary vascular disease in ventricular septal defect: structure and functional correlations in lung biopsies from 85 patients, with outcome of intracardiac repair. F Pathol 1987;152:157-68

9 Haworth SG, Radley-Smith R, Yacoub M. Lung biopsy findings in transposition of the great arteries with ventricular septal defect: potentially reversible pulmonary vascular disease is not always synonymous with operability. $\mathcal{F} \mathrm{Am}$ Coll Cardiol 1987;9:327-33.

10 Berman W Jr, Whitman V, Pierie WS, Waldhausen JA The development of pulmonary vascular obstructive disease after successful Mustard operation in early infancy. Circulation 1978;58:181-5.

11 Castaneda AR, Trusler GA, Paul MH, Blackstone EH, Kirklin JW. The early results of treatment of simple transposition in the current era. $\mathcal{F}$ Thorac Cardiovasc Surg 1988;95:14-28.

12 Mair DD, Ritter DG. Factors influencing intercirculatory mixing in patients with complete transposition of the great arteries. $A m \mathcal{F}$ Cardiol 1972;30:653-8.

13 Zavanella C, Subramaniam S. Review: surgery for transposition of the great arteries in the first year of life. Ann Surg 1978;187:143-50.

14 Levin DL, Fixler DE, Morriss FC, Tyson J. Morphological analysis of pulmonary vascular bed in infants exposed in utero to prostaglandin synthetase infants exposed in utero to prosta.
inhibitors. $\mathcal{F}$ Pediatr 1978;92:478-83.

15 McKenzie S, Haworth SG. Occlusion of peripheral pulmonary bed in a baby with idiopathic persistent fetal circulation. Br Heart $\mathcal{F} 1981 ; 46: 675-8$. 